\title{
Influence of ARVs on Some Biochemical Changes in Liver Non Enzymatic Markers of HIV Positive Patients Attending Specialist Hospital Sokoto, Nigeria.
}

\author{
${ }^{* 1}$ M.G. Abubakar, ${ }^{1}$ M.M. Abduljalil, ${ }^{1}$ G. Bola-Alaka, ${ }^{2}$ Y.I. Nasiru \\ 1Department of Biochemistry, Usmanu Danfodiyo University, Sokoto, Nigeria \\ ${ }^{2}$ Sokoto State Agency for the Control of AIDS, Tuberculosis and Leprosy (SOSACAT), Sokoto, Nigeria \\ [* Corresponding Author: E-Mail:magusau@hotmail.com, : : +2348035052912]
}

\begin{abstract}
Both HIV infection and antiretroviral drugs (ARVs) are associated with abnormalities of liver function, revealed by both enzymatic and non-enzymatic markers. This study evaluated the effect of HIV infection and antiretroviral drugs on the liver non enzymatic marker (total and direct bilirubin, total protein and albumin level) of HIV positive patients (pre-highly active antiretroviral therapy and those on therapies) attending the voluntary counselling and testing units in the state. Seventy five subjects were enrolled into the study, which constituted 25 HIV negative individuals (control group), 25 HIV positive patient not on antiretroviral therapy and 25 on the therapy. Bilirubin, albumin, total protein and CD4 cell count were determined using standard methods. Significantly $(p<0.05)$ higher level of total bilirubin, direct bilirubin and total protein in the HIV positive non-treated with ARVs was observed compared to the control group. At the initiation of antiretroviral therapy the levels of the three parameters were lower, though statistically not significant except for total protein. Albumin level and CD4 cell count were significantly $(p<0.05)$ lower in the non-treated group compared to the control subjects. But at the initiation of therapy the serum levels of Albumin and $\mathrm{CD}_{4}$ count was significantly $(p<0.05)$ higher except for albumin level. HIV infection lowers the level of liver non enzymatic markers, which increase at the initiation of antiretroviral therapy.
\end{abstract}

Keywords: Bilirubin, Albumin, Total protein and $\mathrm{CD}_{4}$ cell.

\section{INRODUCTION}

Hepatotoxicity is the most frequent $(30 \%)$ toxicity of antiretroviral drugs, which has appeared as one of the leading causes of HIV related illness, death and treatment withdrawal (Nunez et al., 2006). The extensive use of antiretroviral drugs by HIV positive patients as well as the new antiretroviral medications resulted in negative impacts on the clinical outcome of the patients (Palellaet al., 2006).

Abnormalities of liver function are frequent in HIV positive subjects and may be caused by other agents beside HIV itself; others may include: hepatitis viruses (major cause of death to the patients), systemic opportunistic infections, malignancies and ARVs induced hepatotoxicity (Kreisberg, 1995). The hepatotoxicity caused by antiretroviral drugs (ARVs) may be related to all the agents of the ARVs classes (Nickolas and Douglas, 2003). Severe and life threatening hepatotoxicity and fatal fulminant hepatitis have been reported in HIV positive patient taking mono therapy of antiretroviral drugs (Deniz and Tansu, 2002). The NRTIs (the back bone of ARVs) has severe hepatotoxic effect particularly zidovudine mono therapy, which subsequently increases the risk during combination therapy (Nickolas and Douglas, 2003). For the NNRTIs, the hepatotoxicity is commonly seen with nevirapine therapy which typically occurs after 4-5 months (Martínez et al., 2001). Hepatotoxicity is also a well known adverse effect of protease inhibitors with ritonavir being the most frequent cause of hepatotoxicity (Nickolas and Douglas, 2003).

The severity of the liver disease may range from the absence of symptoms to liver decomposition, and the outcome can range from spontaneous resolution to liver failure and death (Clark et al., 2002 and Kramer et al., 2005). The severity of hepatotoxicity in HIV positive patients that $2 \%$ of patients are having acute hepatic necrosis and are dying from liver disease (Reisler et al., 2003).

After the commencement of HAART, the reported incidence of severe liver toxicity ranges from 2 to $18 \%$ (Rodríguez-Rosado et al., 1998 and Servoss et al., 2006). Liver failure is the major leading cause of death among individuals being treated with antiretroviral drugs (Alive and Well, 2002). Other risk of liver disease includes alcohol consumption, because alcohol is a known hepatotoxin and its use has been associated with an increased risk of liver dysfunction (Nuñez et al., 2006). Furthermore, cocaine is one of the causative agents of hepatotoxicity via a toxic oxidative metabolite, which induces mitochondrial damage 
(Campos et al., 2002). Other risk factors associated with liver disease include old age (Campos et al., 2002), female gender (Martín-Carbonero et al., 2003), first exposure to antiretroviral treatment (Wit et al., 2002) and significant $\mathrm{CD}_{4}$ cell gains following HAART initiation (Sulkowski et al., 2002).

Being antiretroviral drugs the only mainstay for treatment of HIV infection as well as it's widely reported hepatotoxic effect, we assessed the effect of the both HIV infection and antiretroviral drugs on liver non enzymatic markers of HIV positive patients attending the ART centre of Specialist Hospital, Sokoto, Nigeria.

\section{MATERIALS AND METHODS Experimental Design}

The study was conducted at antiretroviral therapy centre (ART) of Specialist Hospital, Sokoto, Nigeria. A total of 75 patients were enrolled into the study, which comprised 25 HIV negative subjects (blood donor and control), 25 HIV positive non treated with ARVs and 25 patients treated with ARVs. Inclusion criterion for the study includes: HIV-positive patients not on treatment and those on treatment for one year or less, individuals between the ages of 15-60 year old, individuals with no physical sign of metabolic syndrome and patient who are hepatitis $(A, B$, or $C)$ negative.

\section{Ethical Clearance}

Ethical clearance was obtained from the research and ethical committee of Specialist Hospital, Sokoto and all patients were informed using a standard informed consent form.

\section{Method used in assessment of parameters}

Bilirubin determination: The colorimetric method of Jendrassik and Gorf (1938) was adopted. Bilirubin reacts with diazotized sulphanilic acid in alkaine medium to form a blue coloured complex. Bilirubin is determined in the presence of caffeine which releases albumin bound bilirubin by reacting with diazotized sulphanilic acid.

Albumin determination: The dye binding technique utilizing bromocresol green (BCG) as modified by Biggs and Doumas (1971) as employed. Albumin binds the indicator bromocresol (BCG) in an acidic medium resulting in the formation of a blue green albumin BCG complex. The coloured complex produced is proportional to the concentration of albumin in the serum.

Total Protein determination: This was determined using the method of Doumas (1975). Cupric ions, in an alkaline medium react with the peptide bonds of protein molecules, forming a blue violet coloured complex. The intensity of coloured complex produced is proportional to the amount of protein present in the serum.

$\mathrm{CD}_{4}$ Cell Count: BD FACS count system automated analysis was used in accordance with the method of Cassenset al. (2004).

\section{Analysis}

Graph Instat pad version 3.02 (Graph pad Corp., San Diego, USA) was used for statistical analysis. Descriptive statistics and analysis of variance (Benferroni compare all columns) was used to test for the level of significance between mean. A P value < 0.05 was taken as statistically significant.

\section{RESULTS}

In all the two groups of HIV positive subjects, the result show that most of the patients are at the age of 20 to 39 year having $64 \%$ and $84 \%$ in the non-treated group and treated subjects respectively. Furthermore, $28 \%$ and $12 \%$ of the HIV positive patients were within $40-59$ years of age as shown in the non-treated and treated subject respectively (Table 1).

In the HIV positive patient, $56 \%$ and $64 \%$ in the nontreated and treated groups respectively were females. Males constituted $44 \%$ in the non-treated group and $36 \%$ in the treated group (Table 1).

The result further shows that $52 \%$ and $48 \%$ of the females in the non-treated and treated subjects respectively were mostly housewives (married) followed by single (16\% and $32 \%)$ and widows $(20 \%$ and $12 \%$ ) in the non-treated and treated group respectively.

In the HIV positive non-treated with antiretroviral drugs, $56 \%$ of the patients were in clinical stage I of the infection followed by $28 \%$ in the stage II. For the treated subjects, majority of the patients (44\%) enrolled in to the study were in the stage $\|$ of the disease, followed by $36 \%$ been in the clinical stage I (Table 1). The total protein, total bilirubin, direct bilirubin of all the 25 HIV positive patients non-treated with antiretroviral drugs were significantly $(P<0.05)$ higher than the 
control group. However, in the treated subjects the level of total bilirubin, direct bilirubin and total protein were lower compared to the non-treated group, though the increase is statistically insignificant $(P<0.05)$ except for the total protein. Albumin levels and $\mathrm{CD}_{4}$ cell count of the non-treated subjects, show a significantly lower $(p<0.05)$ compared to the control group. But at the initiation of antiretroviral therapy, the level of albumin and $C_{4}$ cell count in the treated subject were higher though not statistically $(P<0.05)$ significant except for the $\mathrm{CD}_{4}$ cell count (Table 2).

Table 1: Demographic Information of the Study Subjects in Sokoto.

\begin{tabular}{llll}
\hline Parameters & Control & HIV+ Non-treated & HIV+ Treated \\
\hline Age (years) & & & \\
$1-19$ & $1(4 \%)$ & $2(8 \%)$ & $1(4 \%)$ \\
$20-39$ & $16(64 \%)$ & $16(64 \%)$ & $21(84 \%)$ \\
$40-59$ & $8(32 \%)$ & $7(28 \%)$ & $3(12 \%)$ \\
Gender & $20(80 \%)$ & $11(44 \%)$ & $9(36 \%)$ \\
Male & $5(20 \%)$ & $14(56 \%)$ & $16(64 \%)$ \\
Female & $15(60 \%)$ & $4(16 \%)$ & $8(32 \%)$ \\
Marital Status & $5(20 \%)$ & $13(52 \%)$ & $12(48 \%)$ \\
Single & $3(12 \%)$ & $3(12 \%)$ & $2(8 \%)$ \\
Married & $2(8 \%)$ & $5(20 \%)$ & $3(12 \%)$ \\
Divorced & - & $14(56 \%)$ & \\
Widowed & - & $7(28 \%)$ & $9(36 \%)$ \\
Clinical Stage & - & $2(8 \%)$ & $11(44 \%)$ \\
Stage I & - & $1(4 \%)$ & $3(12 \%)$ \\
Stage II & & & $2(8 \%)$ \\
Stage III & Stage IV & &
\end{tabular}

KEY: $n=$ Sample size (25 per group).

Table 2: Biochemical Effect of HIV and ARVs on Liver non Enzymatic Indices and $\mathrm{CD}_{4}$ Cell Count of Control, HIV Positive non-treated and HIV Positive treated used in the Study.

\begin{tabular}{|c|c|c|c|}
\hline Parameters & Control & HIV+ Non-treated & HIV+ Treated \\
\hline T-Bilirubin (mg/dl) & $0.56 \pm 0.04^{*}$ & $0.79 \pm 0.04^{*}$ & $0.69 \pm 0.04$ \\
\hline D-Bilirubin (mg/dl) & $0.27 \pm 0.02^{*}$ & $0.43 \pm 0.01^{*}$ & $0.39 \pm 0.01$ \\
\hline T-Protein (g/dl) & $6.35 \pm 0.16^{*}$ & $8.75 \pm 0.15^{\star a}$ & $6.95 \pm 0.28^{* a}$ \\
\hline Albumin (g/L) & $9.93 \pm 1.91^{*}$ & $27.55 \pm 2.47^{\star}$ & $30.40 \pm 0.89$ \\
\hline $\mathrm{CD}_{4}$ Cells (cell/ $/ \mu \mathrm{L}$ ) & $965.76 \pm 25.93^{*}$ & $308.88 \pm 25.07^{\star a}$ & $442.08 \pm 49.43^{a}$ \\
\hline
\end{tabular}

KEY: $n=$ Sample size (25 per group), Values are expressed as Mean \pm SEM;

Values with the same superscript in column are statistically significant $(P<0.05)$;

D-Bilirubin: Direct Bilirubin, T-Bilirubin: Total Biliburin, T-Protein: Total Protein.

\section{DISCUSSION}

In the current study, the effects of HIV infection and antiretroviral drugs (ARVs) on liver non enzymatic markers which include total and direct bilirubin, total protein, albumin and $\mathrm{CD}_{4}$ cell count were investigated. Majority of the HIV patients were between the age of 20 to 39 years old, constituting $64 \%$ and $84 \%$ of the HIV positive non-treated group and HIV positive treated subjects respectively. Many factors may contribute to the higher HIV infection in the middle age people among which include their physical strength and high sexual activity which is major route for the HIV transmission. The finding also agrees with survey by Federal Ministry of Health, Nigeria (2003) and UNAIDS (2004).

Females form the majority of HIV patients, constituting $56 \%$ and $64 \%$ of HIV positive non-treated and treated subjects respectively. This may be due to practiced of some traditional practices like female genital mutilation (FGM) and having multiple sex partners as may occur in polygamous relationships of paramount importance is 
the lack of western knowledge, in addition to other socioeconomic factors like increase in the commercialization of sex $(\mathrm{FMOH}, 2003)$. Survey by UNAIDS (2010), show that the rate of women infected with HIV rose from $43 \%$ in 1999 to $50 \%$ in 2010 . The report further revealed that in Sub-Saharan Africa, women comprised $59 \%$ of the adult living with HIV infection in 2010. Moreover, another study conducted by the Federal Ministry of Health (FMOH) in 2003 and 2005 reported that in northern Nigeria over $40 \%$ of the married females are on polygamous system of marriage with also over $24 \%$ of males on the same marriage system. In 2010 National HIV sero-prevalence sentinel survey by the FMOH reported that majority of HIV infected people in Nigeria were women. The high practice of polygamous marriage in the state may be the core factor for the high rate of infection among women.

Our results further reveal that $52 \%$ and $48 \%$ of the HIV positive non-treated group and treated subject respectively were married. The result was contrary to a 2010 sentinel survey report by the $\mathrm{FMOH}$ which showed that widows have the highest rate $(6.9 \%)$ followed by single $(5.6 \%)$ and married with $4.9 \%(\mathrm{FMOH}, 2010)$. The possible reason for this may be due to the fact that most of the men in the state possess more than one wife and consequently one infected husband is likely to infect two or more of his wives. Before the introduction of antiretroviral therapy most of the infected men die with the infection leaving their spouse (s) behind, hence the high number of widows than married. With the introduction of ARVs, the rate of death has drastically reduced, which makes the rate of married men and women higher than the widows.

Our study show an significant $(P<0.05)$ increase in serum level of total, direct bilirubin and total protein in HIV positive patients not on treatment when compared with treated.

High level of bilirubin in the blood may be as a result of either the body system is producing excess bilirubin or the liver is unable to eliminate the excess bilirubin produced by the system (Naik, 2010). An elevated level of bilirubin in the blood of HIV positive patients may indicate liver disease due to HIV infection or antiretroviral drugs (Onwuliri, 2004).

The finding of this study was in line with a study by Onwuliri (2004) who reported that total and unconjugated bilirubin concentration were significantly higher in HIV infected subjects compared to control. Another study by Ayelagbe et al. (2014) reported significant increase in the level of total bilirubin in HAART naive subject compared to control group.

Many documented studies revealed that antiretroviral drugs induce liver damage through an elevation in the level of bilirubin. Our finding do not support these claims, as the levels decreased in treated subjects when compared with non treated group, though the increase is not statistically significant. The result of this finding was also contrary to the study by Ayelagbe et al. (2014) as reported significant increase in total and conjugated bilirubin of HIV positive on HAART subjects compared to HAART naïve subjects.

HIV infection is associated with remarkable alteration in the levels of proteins in the liver (Treitinger et al., 2001). Protein abnormalities have been reported in all the stages of HIV infection (Grunfeld et al., 1992 and Jaboor et al., 1999). Jaboor et al. (1999) reported that acute phase response to infection is associated with an increase in protein turnover and remarkable degradation of protein. HIV infection induces leukocyte proliferation, increase in the synthesis of cytokines and immunoglobulin which was shown to contribute to protein turnover (Fleck, 1989).

Fuhrman et al. (2004) and Banh (2006) reported that serum total protein in HIV positive patients decreases during inflammation, which returns to normal level after resolution. Banh (2006) reported that the level of protein can be affected by factors like capillary permeability and drugs (like ARVs).

Furthermore, the result of this finding show that albumin level and $\mathrm{CD}_{4}$ T-cell count of HIV positive non-treated with ARVs has significantly $(p<0.05)$ decreased when compared to the control group. Comparing the level of these parameters of the non-treated group and treated subjects, the result revealed an increase in the level of albumin and $\mathrm{CD}_{4}$ cell count in the treated group compared to non-treated group, though only for $\mathrm{CD}_{4}$ cell count was found to be statistically significant $(p<0.05)$.

Albumin synthesized by the liver helps maintain the amount of blood in the vein and arteries. Decrease in albumin level (hypoalbuminemia) in HIV positive patients may be due to increase in transcapillary escape rate and elevated catabolic rate against 
decrease in hepatic synthesis caused by HIV infection (Fleck et al., 1989).

Immune system activation as a result of HIV infection leads to inflammation (either acute or chronic) in HIV infected patients, which in turn lowers the levels of albumin through imposing liver to channels it to other proteins needed for immune response (Naik, 2010). Both HIV infection and antiretroviral drugs cause kidney disease, cardiovascular disease, and arthritis, which are potential source of chronic inflammation in people living with HIV infection (Fuhrman et al., 2004).

\section{CONCLUSION}

Total bilirubin, direct bilirubin and total protein are significantly $(P<0.05)$ higher in the HIV positive nontreated with ARVs compared to the control group. At the commencement of antiretroviral therapy the levels of these three parameters were lower, though statistically not significant $(P<0.05)$ except for total protein. Albumin level and $C_{4}$ cell count were significantly $(p<0.05)$ lower in the non-treated group compared to the control subject. But at the beginning of the therapy they serum levels of Albumin and $\mathrm{CD}_{4}$ cell count were significantly $(p<0.05)$ higher except for albumin level. HIV infection lowers the level of liver non enzymatic markers, which antiretroviral drugs increase.

\section{REFERENCES}

Alive, A. and Well, M. (2002). AIDS patients killed by antiretroviral drugs not HIV, AIDS Conference in Barcelona, University of Pittsburgh, Pp. 1-3.

Ameh, N. and Abdul, M.A. (2004). Prevalence of domestic violence among pregnant women in Zaria, Nigeria. Annals of African Medicine, 2(1): 46.

Ayelagbe, O.G., Akerele, O.P., Onuegbu, A.J. and Oparinde, D.P. (2014). Drug hepatotoxicity in HIV patients on HAART in southwest Nigeria, Journal Dental and Medicine Siencec, 13(5): 67-70.

Banh, L. (October, 2006). Serum proteins as markers of nutrition: what are we treating? Practical Gastroenterology, 46-64.

Biggs, H.G., Doumas, B.T. and Watson, W.A. (1971). Clinical Chemistry, J clin chem, 31:81-87.

Campos, J., Martinez, C., Perez, E. and Gonzalez, A. (2002). Cocaine related fulminant liver failure, Anales de Medicina Interna., 19:365-367.

Cassens, U., Gohde, W., Kuling, G., Gröning, A., Schlenke, P., Lehman, L.G., Traore, Y., Servais, J. and Henin, Y. (2004). Simplified Volumetric Flow
Cytometry allows Feasible and Accurate Determination of $\mathrm{CD}_{4} \mathrm{~T}$ - Lymphocytes in Immunodeficient Patients Worldwide, Antiviral Therapy, 9: 395-405.

Clark, S., Creighton, S., Portmann, B., Taylor, C., Wendon, J. and Cramp, M. (2002). Acute liver failure associated with antiretroviral treatment for HIV: a report of six cases, Journal of Hepatology, 36: 295-301.

Deniz, G., and Tansu, Y. (2002). Hepatic adverse events during highly active antiretroviral therapy containing nevirapine: a case report, Annals of Clinical Microbiology and Antimicrobials 1: 2-8

Doumas, B.T. (1975). Clinical Chemistry, A Journal of Clinical Chemistry, 21: 59-61.

Federal Ministry of Health (2003). National HIV Seroprevalence Sentinel Survey. Abuja: Federal Ministry of Health.

Federal Ministry of Health (2010). National HIV seroprevalence sentinel survey among pregnant women attending ANC, Federal Ministry of Health, Abuja, Nigeria

Fleck, A. (1989). Clinical and nutritional aspects of changes in acute phase proteins during inflammation, Proceedings of the Nutrition Society, 48: $347-54$.

Fuhraman, M.P., Charney P. and Mueller, C.M. (2004). Hepatic proteins and nutrition assessment. Journal of the American Dietetic Association, 104: 12581264.

Grunfeld, C., Pang, M. and Doerrler W. (1992). Lipids, Lipoproteins, Triglyceride clearance and cytokines in HIV infection and the AIDS, The Journal of Clinical Endocrinology \& Metabolism, 74: 10451052.

Jaboor, F., Gazzard, B. and Phillips G. (1999). the acute phase protein response to HIV infection in human subjects, American Journal of Physiology Endocrinology and Metabolism, 276(6): E1092E1098.

Jendressik, L. and Gorf, P. (1938). Clinical Biochemistry, Biochemische Zeitschrift. 297: 8189

Kreisberg, R. (1995). Clinical Problem-solving. We blew it. The New England Journal of Medicine, 332:945949.

Martín-Carbonero, L., Nunez, M., Gonzalez-Lahoz, J. and Soriano, V. (2003). Incidence of liver injury after beginning antiretroviral therapy with efavirenz or nevirapine, HIV Clinical Trials, 4:115-120. 
Martínez, E., Blanco, J. and Arnaiz, J. (2005). Hepatotoxicity in HIV-1 infected patients receiving nevirapine-containing antiretroviral therapy. AIDS, 15:1261-8.

Naik, P. (2010). Biochemistry, $3^{\text {rd }}$ edition, Jaypee Brothers Medical Publishers (P) Itd., India, Pp. 618-622.

Nickolas, K. and Douglas, D. (2003). Hepatotoxicity of Antiretroviral Therapy, AIDS Reviews, 5: 36-40.

Nunez, M.J., Martin-Carbonero, L., Moreno, V., Valencia, E., Garcia-Samaniego, J. and GonzalezCastillo J. (2006). Impact of antiretroviral treatment-related toxicities on hospital admissions in HIV-infected patients, AIDS Research and Human Retroviruses, 22(9): 825-829.

Onwuliri, V.A. (2004). Total Bilirubin, Albumin, Electrolytes and Anion Gap in HIV positive patients in Nigeria, Journal of Medical Sciences, 4(3): 214-220.

Palella, F., Baker, R., Moorman, A., Chmiel, J., Wood, K. and Brooks, J. (2006). Mortality in the highly active antiretroviral therapy era: changing causes of death and disease in the HIV outpatient study, Journal of Acquired Immune Deficiency Syndromes, 43: 27-34.

Reisler, R., Han, C., Burman, W., Tedaldi, E. and Neaton J. (2003). Grade 4 events are as important as AIDS events in the era of HAART. Acquired Immune Deficiency Syndromes, 34: 379-386.

Rodríguez-Rosado, R., García-Samaniego, J. and Soriano. V. (1998). Hepatotoxicity after introduction of highly active antiretroviral therapy, AIDS, 12:1256.

Servoss, J., Kitch, D., Andersen, J., Reisler, R., Chung, R. and Robbins G. (2006). Predictors of antiretroviral-related hepatotoxicity in the adult AIDS Clinical Trial Group (1989-1999), Journal of Acquired Immune Deficiency Syndromes, 43: 320323.

Sulkowski, M., Thomas, D., Mehta, S., Chaisson, R. and Moore R. (2002). Hepatotoxicity associated with nevirapine- or efavirenz-containing antiretroviral therapy: role of hepatitis $\mathrm{C}$ and $\mathrm{B}$ infections. Hepatology, 35: 182-189.

Treitinger, A., Spada, C., Mari, L., Silva, D., Martins, E.H., Amaral, J.A. and Abdalla, D.S. (2001). Lipid and Acute Phase Protein Alteration in HIV-1 Infected Patients in the Early Stages of Infection: Correlation with $\mathrm{CD}_{4}$ lymphocytes, The Brazilain Journal of Infectious Diseases, 5(4): 192-199.

UNAIDS, (2004). Epidemiological fact sheet on HIVIAIDS and sexually transmitted infections, Nigeria, Page 3-11, available at www.unaids.org

UNAIDS, (2010). Global Report on the global AIDS epidemic: Annexes, Joint nations programme on HIVIAIDS (UNAIDS), adapted from www.unaids.org/en/media/unaids/ documents

Wit, F., Weverling, G., Weel, J., Jurrians, S. and Lange J. (2002). Incidence and risk factors for severe hepatotoxicity associated with antiretroviral combination therapy. The Journal of Infectious Diseases, 186: 23-31. 